\title{
CANCER
}

\section{Regression analysis of trends in mortality from hepatocellular carcinoma in Japan, 1972-2001}

\author{
Kenji Shibuya $^{1,2 *}$ and Eiji Yano ${ }^{2}$
}

\begin{abstract}
Accepted 15 September 2004
Background In Japan, higher mortality rates from hepatocellular carcinoma (HCC) have been observed than in any other industrialized country and the reported numbers of deaths due to HCC have increased significantly in recent years. We assessed whether there was a real increase in mortality from HCC and which factors contributed to the recent increasing trends of the number of deaths from HCC.
\end{abstract}

Methods Poisson regression models were used to analyse panel data for the period 1972-2001.

Results In both sexes, age-standardized mortality rates from HCC increased significantly over the past three decades. Among males the annual percentage increase in ageadjusted mortality rates was approximately 1-3\% during the period 1972-96. On the other hand, female mortality trends were less clear. The estimated birth cohort effects suggested that there was a peak in the mortality risk among the cohorts born during the period 1927-36, which corresponded to those aged between 50-64 years during the period 1987-96. This was the period when a large increase of both age-specific mortality rates among older age groups and age-standardized mortality rates were observed particularly among males.

Conclusions This study shows that an upward trend of mortality from HCC in Japan was primarily due to the effects among birth cohorts born around 1930, which are consistent with the pattern of past exposure to hepatitis $\mathrm{C}$ virus. The cohort effects have contributed to a large increase in mortality from HCC in recent years and the number of deaths from HCC is expected to increase by 2010-15.

Keywords Hepatocellular carcinoma, mortality, trends, cohort effects, hepatitis C virus

Over the past decade, increase in the number of cases of hepatocellular carcinoma (HCC) as well as deaths from it have been reported in industrialized countries, including Japan, the United States, and some European countries. ${ }^{1-3}$ In Japan, higher mortality rates have been observed than in any other industrialized country and the reported numbers of deaths due to HCC have increased significantly in recent years. ${ }^{4}$ In Japan

\footnotetext{
${ }^{1}$ Measurement and Health Information Systems, World Health Organization, Geneva, Switzerland.

2 Department of Hygiene and Public Health, Teikyo University School of Medicine, Tokyo, Japan.

* Corresponding author. Measurement and Health Information Systems, World Health Organization CH-1211, Geneva 27, Switzerland. E-mail: shibuyak@who.int
}

HCC is now the third and fourth largest cause of cancer mortality among males and females, respectively. ${ }^{5}$

However, several factors have an influence on the secular mortality trends other than a real increase in mortality from HCC. These include changes in classification systems and coding practices, diagnostic techniques and ascertainment, age distribution, and survival from the disease. ${ }^{1,2,6-8}$ Although monitoring trends of mortality can allow potential factors, which influence both incidence and mortality, to be determined, no published studies have analysed these factors in relation to the trends of mortality from HCC in Japan. The purpose of this study is to assess whether there was a real increase in mortality from HCC and the factors that contributed to an increasing trend in mortality from HCC during the past three decades in Japan. 


\section{Material and Methods \\ Data sources}

Vital statistics data on the numbers of deaths from hepatocellular carcinoma (HCC) and the estimates on agespecific mid-year population for each calendar year from 1972 to 2001 were used for the analyses. Each death was characterized by detailed demographic data including age at death, year of death, and year of birth. We used the data on mortality coded as 1550 for the period 1972-1994 (ICD-8 and 9) and C220 (ICD-10) for the period 1995-2001. In order to make a comparison, we also analysed mortality from liver cirrhosis (coded as 571 in the ICD-8 and 9, and K70 and 74 in the ICD-10). The minimum age group for this study was 20-24 years. For men and women separately, the number of deaths and populations were stratified by five-year age groups $(20-24, \ldots, 85-89)$, five-year calendar periods $(1972-76, \ldots$, 1997-2001), and five-year birth cohorts (1882-86,..., 1972-76). Data on incidence of post-transfusion hepatitis and prevalence of hepatitis virus infections for the study periods were obtained from the Japanese Red Cross.

\section{Statistical analysis}

Trends in mortality can be characterized by one or more of three types of effects: age, period, and cohort. ${ }^{9,10}$ Age effects are present for virtually every cause of death. Period effects typically occur as a result of changes affecting all age groupsfor example, changes in diagnostic standards and cure rates, and introduction of an aetiologic agent with a rapid effect. Cohort effects typically occur when people born at a given time are more exposed to an agent as compared with people born at another time.

Age- and sex-specific mortality rates were calculated and tested for trends. ${ }^{11}$ We then carried out two types of regression analysis. First, to assess both overall trends and trends within each 5-year period, we estimated age-standardized rate and annual percentage change for each 5 -year period. We employed a log-linear Poisson regression model to estimate annual percentage change, in which age groups and calendar years were included as independent variables:

$$
\ln \left(\frac{D_{i j}}{N_{i j}}\right)=\alpha_{i} A G E_{i}+\beta_{j} \text { PERIOD }_{j}+\varepsilon_{i j}
$$

where $D_{i j}$ and $N_{i j}$ are, respectively, the number of deaths and the size of the population in age group $i$ in time period $j$, and $\alpha_{i}$ represents the effect of age group $i$ and $\beta_{j}$ the effect of time period $j$. Second, despite its popularity in the assessment of chronic diseases, separate effects due to age, period, and cohort for mortality rates of a particular disease can hardly be quantified by a three-factor multiplicative model (i.e. ageperiod-cohort model) because of the non-identifiability problem arising from the linear relationship among the three factors. ${ }^{12-14}$ Possible solutions have been proposed, including the use of individual-level data, the application of other covariates in place of either period or cohort variable, and some mathematical constraints to a model. ${ }^{14}$ In the present study, we employed the first two approaches to avoid the problem. Since the trends in liver cancer morbidity and mortality are strongly related to changes in hepatitis infection, ${ }^{15,16}$ we used the incidence of post-transfusion hepatitis as a covariate in place of the period variable in the second Poisson regression model: ${ }^{17}$

$$
\ln \left(\frac{D_{i j k}}{N_{i j k}}\right)=\alpha_{i} A G E_{i}+\beta P R E V_{j}+\gamma_{k} \text { COHORT }_{k}+\varepsilon_{i j k}
$$

where $P R E V_{j}$ denotes the continuous variable of incidence of post-transfusion hepatitis in time period $j$ and $\gamma_{k}$ denotes the effect of birth cohort $k$. Both models were then fit to the data by maximum likelihood methods. Person-years at risk were approximated by the size of the mid-year population in each year.

\section{Results}

Figure 1 represents age- and sex- specific mortality rates of hepatocellular carcinoma (HCC) from 1972 to 2001. There was a statistically significant increase in mortality rates among males aged 65 and over. On the contrary, mortality rates in younger age groups have started to decrease since the 1980s. Female mortality rates showed a less clear trend, but a steady increase in mortality among older age groups was also observed since 1992-96.

In both sexes, the number of deaths from HCC increased substantially during the past three decades and the mortality from HCC among men almost tripled (Table 1). Overall trends of age-specific mortality rates are summarized by agestandardized mortality rates and annual percentage changes in mortality. Male mortality rates, age-standardized to the 1990 population, increased by $70 \%$ over the past two decades from 17.4 per 100000 in $1972-76$ to 29.6 per 100000 in 1992-96, but decreased slightly in the subsequent period. On the other hand, female age-standardized mortality from HCC showed a slight increase during the past three decades. Mortality rates for liver cirrhosis showed a substantial decline in both sexes since the 1970s: male and female mortality rates decreased from 27.0 and 10.9 per 100000 in $1972-76$ to 9.8 and 4.2 per 100000 in 1997-2001, respectively. The trends of age-standardized mortality rates were confirmed with the annual percentage changes in mortality rates. Among males, the annual percentage change in age-adjusted mortality rates of HCC was approximately 1-4\% through the period 1972-96 with a subsequent decline in 1997-2001. On the other hand, the change among females was not significant, except for a substantial increase in the early 1990s and with a subsequent decline in 1997-2001. Both absolute and percentage changes of mortality were not parallel for HCC and liver cirrhosis in both sexes during the entire period from 1972 to 2001.

After adjusting for age and period effects, the cohort effect of mortality risk from HCC is estimated from Equation (2). For example, the risk of mortality among birth cohorts born in 1952-56 relative to the reference cohort born in 1882-86 is: $\exp \left(\gamma_{1952-56}\right) / \exp \left(\gamma_{1882-86}\right)=\exp \left(\gamma_{1952-56}-1\right)$. Figure 2 shows the estimated relative risks of mortality from HCC for each birth cohort in a logscale. In both sexes, there was a peak in mortality risk for the cohorts born during 1927-36, which corresponded to those aged 50-64 years in 1987-91 and those aged 5569 years in 1992-96, respectively. This increasing trend in cohort effects before the mid-1930s is consistent with a large increase of both age-specific mortality rates among older age groups and 

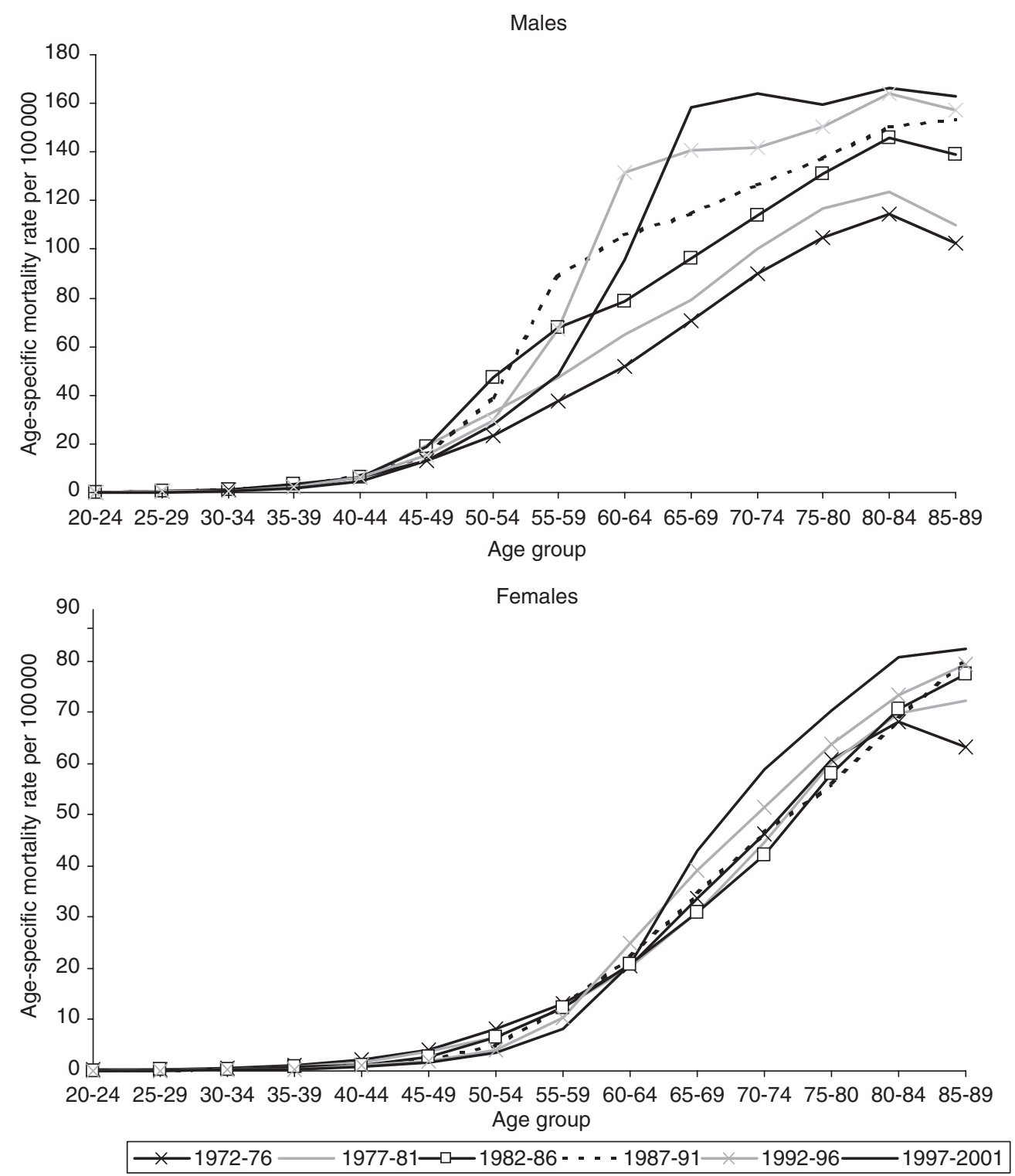

Figure 1 Age-specific mortality rates of hepatocellular carcinoma in Japan, 1972-2001

age-standardized mortality rates for the period 1986-96, in particular among males. The levels of mortality risks in both sexes levelled off and continued to decline for the cohorts born thereafter and the relative risk of HCC mortality returned to the levels before the study period among those born after 1955 .

\section{Discussion}

HCC is one of the most common malignancies in the world, predominantly affecting the developing regions where the hepatitis virus is endemic. ${ }^{18}$ Over the past two decades, however, an increase in mortality and morbidity from HCC has been observed in industrialized countries. ${ }^{1-3}$ The numbers of deaths due to HCC increased significantly in Japan over the past three decades, from 51000 to 153000 , and its age-standardized mortality rate of HCC is the highest among industrialized countries. ${ }^{19}$
It should be noted, however, that several factors other than changes in actual mortality have an influence on the secular trends of HCC mortality, including changes in: classification and coding, diagnostic techniques and ascertainment, age distribution, and survival from the disease. ${ }^{1,2,6-8}$ Although primary liver cancer may be recorded in the mortality statistics rather than a definitive diagnosis HCC or hepatoblastoma in the eighth and ninth revisions of the International Classification of Diseases and Related Health Problems (ICD), the vast majority of primary liver cancer in Japan is HCC and the proportion of hepatoblastoma and misclassified metastatic liver tumours is very small. ${ }^{16}$ In addition, age-standardized rates of primary liver cancer did not change according to the revision of ICD classification systems. Thus, the effects of any changes in coding and the disease classification system are likely to have been very small and unable to bias seriously the trends over time. 
Table 1 Mortality from hepatocellular carcinoma and liver cirrhosis in Japan, 1972-2001

\begin{tabular}{|c|c|c|c|c|c|c|}
\hline \multirow[b]{2}{*}{ Period } & \multicolumn{3}{|c|}{ Hepatocellular carcinoma } & \multicolumn{3}{|l|}{ Liver cirrhosis } \\
\hline & $\begin{array}{c}\text { Age-standardized } \\
\text { mortality rate }\end{array}$ & $\begin{array}{l}\text { Annual percentage } \\
\text { change }(95 \% \mathrm{CI})\end{array}$ & $\begin{array}{r}\text { Total number } \\
\text { of deaths }\end{array}$ & $\begin{array}{l}\text { Age-standardized } \\
\text { mortality rate }\end{array}$ & $\begin{array}{r}\text { Annual percentage } \\
\text { change }(95 \% \mathrm{CI})\end{array}$ & $\begin{array}{r}\text { Total number } \\
\text { of deaths }\end{array}$ \\
\hline \multicolumn{7}{|l|}{ Males } \\
\hline $1972-76$ & 17.4 & $1.8^{\mathrm{b}}(1.0-2.5)$ & 32335 & 27.0 & $0.7^{\mathrm{b}}(0.1-1.3)$ & 52428 \\
\hline 1977-81 & 20.7 & $4.2^{\mathrm{b}}(3.5-4.9)$ & 45176 & 25.9 & $-1.2^{\mathrm{b}}(-1.8$ to -0.6$)$ & 58472 \\
\hline $1982-86$ & 25.3 & $3.3^{\mathrm{b}}(2.7-3.9)$ & 63966 & 23.0 & $-2.8^{\mathrm{b}}(-3.4$ to -2.3$)$ & 59240 \\
\hline $1987-91$ & 28.5 & $1.1^{\mathrm{b}}(0.6-1.6)$ & 83485 & 19.4 & $-2.4^{\mathrm{b}}(-3.0$ to -1.8$)$ & 56999 \\
\hline 1992-96 & 29.6 & $1.6^{\mathrm{b}}(1.1-2.0)$ & 100560 & 15.6 & $-9.6^{\mathrm{b}}(-10.2$ to -9.1$)$ & 51348 \\
\hline $1997-2001$ & 27.4 & $-2.7^{\mathrm{b}}(-3.1$ to -2.3$)$ & 109365 & 9.8 & $-11.4^{\mathrm{b}}(-12.1$ to -10.8$)$ & 36174 \\
\hline \multicolumn{7}{|l|}{ Females } \\
\hline $1972-76$ & 10.0 & $-2.4^{\mathrm{b}}(-3.4$ to -1.4$)$ & 18167 & 10.9 & $-2.1^{\mathrm{b}}(-3.1$ to -1.2$)$ & 20229 \\
\hline $1977-81$ & 9.6 & $-0.6(-1.6$ to 0.4$)$ & 20602 & 10.1 & $-1.0^{\mathrm{b}}(-1.9$ to 0.0$)$ & 22039 \\
\hline $1982-86$ & 9.4 & $0.1(-0.9$ to 1.0$)$ & 23960 & 9.5 & $-1.0^{\mathrm{b}}(-1.9$ to -0.1$)$ & 24452 \\
\hline 1987-91 & 9.6 & $0.8(-0.1$ to 1.6$)$ & 28935 & 8.6 & $-1.7^{\mathrm{b}}(-2.6$ to -0.8$)$ & 25976 \\
\hline $1992-96$ & 10.2 & $3.0^{\mathrm{b}}(-2.3$ to 3.8$)$ & 36331 & 6.7 & $-12.1^{\mathrm{b}}(-12.9$ to -11.3$)$ & 23319 \\
\hline $1997-2001$ & 10.6 & $-0.8^{\mathrm{b}}(-1.4$ to -0.1$)$ & 43871 & 4.2 & $-6.1^{\mathrm{b}}(-7.1$ to -5.1$)$ & 16978 \\
\hline
\end{tabular}

${ }^{\mathrm{a}}$ Rates are age-standardized to the 1990 population and per 100000 populations.

${ }^{b_{P}}<0.05$ for the null hypothesis that the annual per cent change is equal to zero.

The changes might have been an artefact of improvements in diagnosis, which would provide a more accurate picture of mortality trends. In particular, substantial improvements in the detection of liver cancer in cirrhotic patients can be expected through the introduction of new diagnostic techniques, and a shift of death certification from cirrhosis to liver cancer would affect HCC mortality significantly. ${ }^{2}$ However, the routine use of ultrasonography and serum alpha-fetoprotein since the early 1980s in cirrhotic patients could not explain continuously increasing trends of mortality from 1982 to 1996. The annual increase in the liver cancer mortality rate and the annual decline in cirrhotic mortality were not parallel for the past three decades in both sexes. Other possible factors would include changes in prognosis of treated HCC. However, the recent studies have consistently shown improvements in overall survival rates of HCC ${ }^{20}$ which contradict currently observed mortality trends.

Although HCC predominantly affects the elderly populations in industrialized countries, ageing alone cannot explain the rising mortality from HCC as shown by the trends of age-specific mortality rates. The rise in age-specific mortality in the older groups was coupled with an initial increase and subsequent reduction in mortality among younger age groups. If the overall mortality were the reflection of an ageing population, one would expect an increase in mortality that is either restricted to or out of proportion, among persons in the older age groups, irrespective of when they were born. ${ }^{1}$ On the other hand this trend is most probably the result of the exposure to risk factors, which affect all birth cohorts but are stronger in cohorts born during a certain period. In fact, the present study has confirmed an upward trend of mortality from HCC in Japan due to the effects of birth cohorts born around the 1930s, which have contributed to a large increase in mortality from HCC in the recent years.

Among the histologically confirmed HCC, the majority of cases were due to hepatitis $\mathrm{C}$ virus as reported by others. ${ }^{16,21}$ Although hepatitis B virus ( $\mathrm{HBV})$ is more common in many parts of Asia, the relative importance of hepatitis C virus (HCV) as a cause of HCC has been significantly increased in Japan after the introduction of HBV screening in 1972 and nearly $90 \%$ of hepatocellular carcinoma is now caused by HCV. ${ }^{5,15,21-23}$ One possible explanation is that the trends of the cohort effects are consistent with the pattern of past exposure to hepatitis viruses. In fact, the cohort effect in both sexes showed a similar pattern, with a peak for the cohorts born during 1927-36. These cohorts corresponded to those aged 50-64 years in 1987-91 and those aged 55-69 years in 1992-96, in which the highest prevalence of HCV among the voluntary blood donors was observed. ${ }^{24-26}$ For example, Tanaka and colleagues investigated 197600 voluntary blood donors aged 15-64 years residing in Osaka in the early 1990s and found that the prevalence of anti-HCV antibody was significantly higher in the blood donors aged 55-64 years than in those aged 1654 years (8.5 vs $1.3 \%$ among males; 7.3 vs $1.4 \%$ among females). ${ }^{25}$ The age pattern of HCV prevalence was consistent with the latest study on 3.5 million pooled blood donors during $1995-2000 .^{26}$ For the cohorts born after the 1950s, the risk of mortality from HCC decreased rapidly and we would expect a further mortality decline due to the introduction of $\mathrm{HCV}$ screening in $1989 .{ }^{24,27}$

The majority of the study populations are the cohorts born before 1950, which were under 50 years of age during the 1950s and 1960s. This was exactly the time when illicit intravenous drug abuse (i.e. meta-amphetamine addiction) was endemic in Japan and, according to the official statistics, more than $5 \%$ of the population aged 15-25 years were drug abusers. $^{28}$ The spread of hepatitis viruses, particularly HCV, from drug abusers to the general population was most likely due to transfusion of unscreened or commercial blood and blood products and medical practices (e.g., needle sharing for immunizations). ${ }^{19,24,29,30}$ In fact, before commercial blood was replaced by screened and voluntarily donated blood in 1969 , the probability of developing post-transfusion hepatitis reached nearly $50 \%$. Since the cohorts born around the 1930s was 


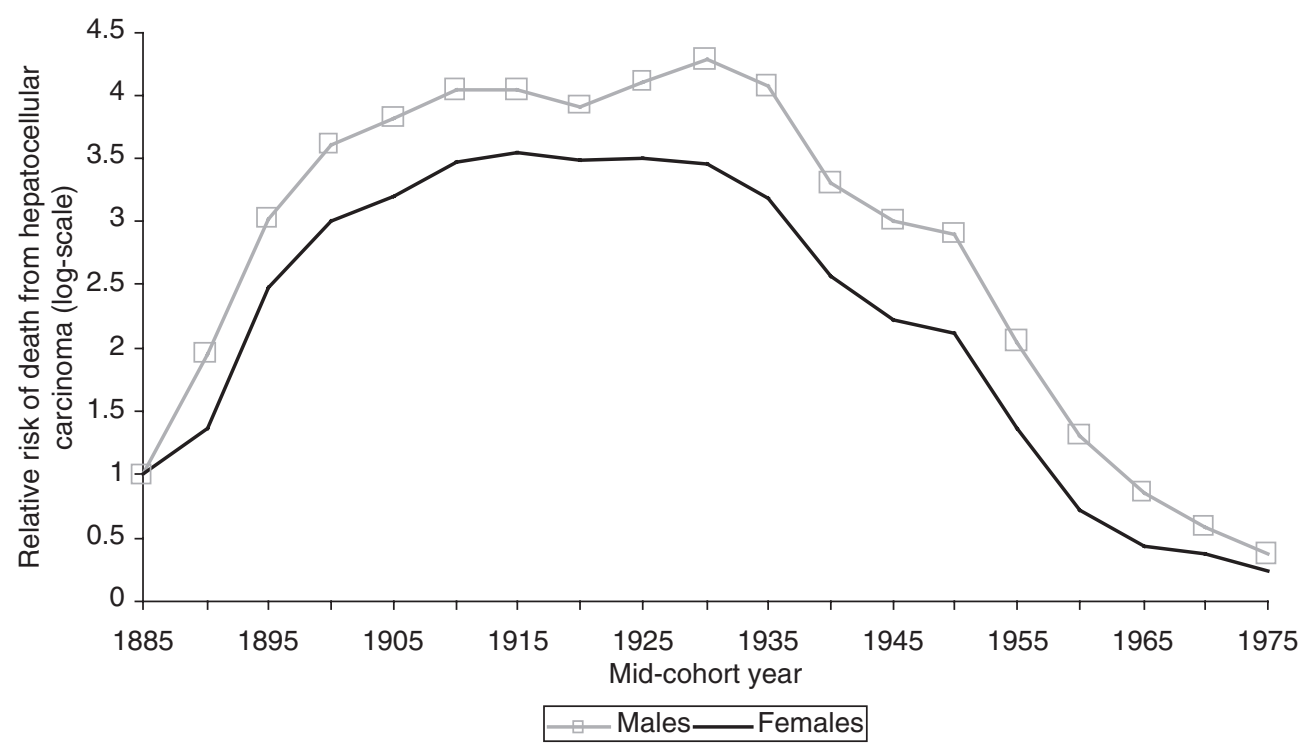

Figure 2 Relative risk of mortality from hepatocellular carcinoma by birth cohort in Japan (in a log-scale, reference birth cohort = 1882-1886)

about 10-30 years of age during the 1950s and 1960s, their risk of becoming infected with hepatitis viruses was high, as suggested by the cohort risk of mortality from HCC. Since there is an approximately 30-year time lag between exposure to hepatitis viruses and liver cancer, ${ }^{31,32}$ those infected with hepatitis viruses during the 1950s or 1960s would develop HCC in the 1980s and 1990s, during which period mortality from HCC rapidly increased in Japan.

Due to the ecological nature of the current analysis, however, the interpretation of the results should be made with caution. ${ }^{33}$ For example, cohort effects would also reflect unobserved factors other than exposure to the HCV. Further studies would be needed to assess the plausibility of the inference made above.

Although the horizontal and vertical transmissions of HBV and of HCV have been substantially reduced since the introduction of blood screening, there are still 2-3 million carriers infected with hepatitis viruses, ${ }^{29}$ the majority of which are infected with HCV. The number of deaths from HCC is expected to increase by 2010-15 since it was only after the cohorts born around 1950 and thereafter that the relative risks of HCC mortality returned to the levels before the study period.

It is suggested that approximately $2-7 \%$ of all patients with chronic HCV will develop HCC. ${ }^{1,34,35}$ Responding to the public awareness of a rapid increase in mortality and morbidity from HCV infection, the Japanese Government has recently implemented the population-based screening programme of anti-HCV antibody in housewives and elderly people outside workforces aged between 40 and 70 years. Further analysis of the future trends of mortality and incidence of HCC and other consequences of chronic HCV infection is required to test whether the programme should be targeted at the general public over the age of 40 or those at a higher risk including haemodialysis and haemophilia patients, and recipients of blood or blood products before 1992..$^{29,30}$

\section{Acknowledgements}

The authors wish to thank Atsushi Koyama for extracting and compiling the data, Gary King for the valuable comments on statistical analysis, and Sue Piccolo for her editorial assistance. This study was supported by the grant from the Pfizer Health Research Foundation, Tokyo, Japan (No. 02A085). The authors certify that they have not entered into any agreement that could interfere with their access to the data on the research, nor upon their ability to analyse the data independently, to prepare articles, and to publish them.

\section{KEY MESSAGES}

- In both sexes, there was a considerable increase in age-standardized mortality rates from hepatocellular carcinoma (HCC) during the period 1972-2001 in Japan.

- An upward trend of mortality from HCC in Japan was primarily due to the effects among birth cohorts born around 1930, which are consistent with the pattern of past exposure to hepatitis C virus.

- Birth cohort effects have contributed to a large increase in mortality from HCC in recent years and the number of deaths from HCC is expected to increase by 2010-15. 


\section{References}

${ }^{1}$ El-Serag HB, Mason AC. Rising incidence of hepatocellular carcinoma in the United States. N Engl J Med 1999;340:745-50.

${ }^{2}$ La Vecchia C, Lucchini F, Franceschi S, Negri E, Levi F. Trends in mortality from primary liver cancer in Europe. Eur $J$ Cancer 2000;36:909-15.

${ }^{3}$ Okuda K, Fujimoto I, Hanai A, Urano Y. Changing incidence of hepatocellular carcinoma in Japan. Cancer Res 1987;47:4967-72.

${ }^{4}$ Easton A. Liver cancer toll high in Japan. BMJ 1999;318:1510.

${ }^{5}$ Kiyosawa K, Tanaka E. Characteristics of hepatocellular carcinoma in Japan. Oncology 2002;62(Suppl. 1):5-7.

${ }^{6}$ Hennekens CH, Buring JE. Epidemiology in Medicine. Boston: Little Brown, 1987.

${ }^{7}$ Kaczynski J, Oden A. The rising incidence of hepatocellular carcinoma. N Engl J Med 1999;341:451.

${ }^{8}$ Stuver S, Trichopoulos D. Liver cancer. Cancer Surv 1994;19-20:99-124.

${ }^{9}$ Clayton D, Schifflers E. Models for temporal variation in cancer rates II: age-period-cohort models. Stat Med 1987;6:469-81.

${ }^{10}$ Holford TR. The estimation of age, period, cohort effects for vital rates. Biometrics 1983;39:311-24.

${ }^{11}$ Kim H, Fay M, Feuer E, Midthune D. Permutation tests for joinpoint regression with applications to cancer rates. Stat Med 2000;19:335-51.

12 Holford TR. Understanding the effects of age, period, and cohort on incidence and mortality rates. Annu Rev Public Health 1991;12:425-57.

${ }^{13}$ Moolgavkar SH, Lee JAH, Stevens RG. Analysis of vital statistics data. In: Rothman KJ, Greenland S (eds). Modern Epidemiology, 2nd (Edn). Philadelphia: Lippincott-Raven, 1998, pp. 481-97.

${ }^{14}$ Robertson C, Boyle P. Age-period-cohort analysis of chronic disease rates I: modelling approach. Stat Med 1998;17:1305-23.

${ }^{15}$ Heintges T, Wands JR. Hepatitis C virus: epidemiology and transmission. Hepatology 1997;26:521-26.

16 The Liver Cancer Study Group. Primary liver cancer in Japan: Sixth report. The Liver Cancer Study Group of Japan. Cancer 1987;60:1400-11.

${ }^{17}$ Rodgers WL. Estimable functions of age, period, and cohort effects. Am Sociol Rev 1982;47:774-78.

18 Murray CJL, Lopez AD. Estimating causes of death: new methods and global and regional applications for 1990. In Murray CJL, Lopez AD (eds). The Global Burden of Disease. Cambridge: Harvard University Press, 1996, pp. 117-200.
${ }^{19}$ Easton A. Liver cancer toll high in Japan. BMJ 1999;318:1510.

${ }^{20}$ Matsumata T, Kanematsu T, Shirabe K, Sonoda T, Furuta T, Sugimachi K. Decaresed morbidity and mortality rates in surgical patients with hepatocellular carcinoma. Br J Surg 1990;77:677-80.

${ }^{21}$ Kubo S, Nishiguchi S, Hirohashi K et al. High prevalence of infection with hepatitis $\mathrm{B}$ and $\mathrm{C}$ viruses in patients with hepatocellular carcinoma in Japan. Hepatogastroenterology 1999;46:357-59.

22 Shiratori Y, Shiina S, Imamura M, Kato N, Kanai F, Okudaira T. Characteristic difference of hepatocellular carcinoma between hepatitis Band C-viral infection in Japan. Hepatology 1995;22:1027-33.

${ }^{23}$ Tanaka K, Hirohata T, Koga S et al. Hepatitis C and hepatitis B in the etiology of hepatocellular carcinoma in the Japanese population. Cancer Res 1991;51:2842-47.

${ }^{24}$ Moriya T, Koyama T, Tanaka J, Mishiro S, Yoshizawa H. Epidemiology of hepatitis C virus in Japan. Intervirology 1999;42:153-58.

25 Tanaka H, Hiyama T, Tsukuma $\mathrm{H}$ et al. Prevalence of second generation antibody to hepatitis $\mathrm{C}$ virus among voluntary blood donors in Osaka. Cancer Causes Control 1994;5:409-13.

${ }^{26}$ Tanaka J, Kumagai J, Katayama K et al. Sex- and age-specific carriers of hepatitis B and C viruses in Japan estimated by the prevalence in the 3,485,648 first-time blood donors during 1995-2000. Intervirology 2004; 47:32-40.

${ }^{27}$ Schreiber GB, Busch MP, Kleinmann SH, Korelitz JJ. The declining risk of transfusion-transmitted viral infections. $N$ Engl J Med 1996;334:1685-90.

28 Japan Ministry of Health and Welfare. Summary of opioids and stimulants abuse. Tokyo, 1990.

29 Japan Society of Hepatology. White book on liver cancer. Tokyo, 1997.

${ }^{30}$ Yoshizawa H. Current status of chronic hepatitis in Japan. Acto Hepatologica Japonica (in Japanese) 2000;41:4-5.

31 Alberti A, Chemello L, Benvegnu L. Natural history of hepatitis C. Hepatology 1999;31(Suppl.1):17-24.

32 Castells L, Vargas V, Gonzalez A, Esteban J. Long interval between HCV infection and the development of hepatocellular carcinoma. Liver 1995;15:159-63.

33 Schwartz S. The fallacy of the ecological fallacy: the potential misuse of a concept and the consequences. Am J Public Health 1994;84:819-24.

${ }^{34}$ Di Bisceglie AM, Rustgi VK, Hoofnagle JH, Dusheiko GM, Lotze MT. NIH conference: hepatocellular carinoma. Ann Intern Med 1988;108:390-401.

${ }^{35}$ Di Bisceglie AM. Hepatitis C and hepatocellular carcinoma. Hepatology 1997;26(Suppl.1):34S-8S 